\title{
RADIOFREQUENCY ABLATION TECHNIQUE FOR DEEP SEATED BRAIN TUMORS
}

\author{
Ahmed Hazem Soliman , Abd El monaem Ismail Mokbel, Mohamed Gouda Ammar, Mohamed \\ Salah Mohamed \\ Department of Neurosurgery, Faculty of Medicine ,Zagazig University, Egypt
}

ABSTRACT

Fifteen pathologically diagnosed patients with grade II,III and IV primary or recurrent glioma (tumor diameter $2-5 \mathrm{~cm}$ ) were randomly selected and underwent hyperthermia treatment in the form of radiofrequency treatment, and patients with recurrent tumors were treated with hyperthermia in combination with radiotherapy and chemotherapy. Hyperthermia treatment was administered at $90^{\circ} \mathrm{C}$ radio-frequency hyperthermia device. Electrodes were inserted into the tumor with the aid of a CT-guided stereotactic apparatus and heat was applied for 5 minutes . During follow up after hyperthermia treatment, patients were evaluated with head CT or MRI every month. Gliomas in the hyperthermia group exhibited growth retardation or growth termination. Decrease in tumour diameter was evident in $85 \%$ of the heated tumor tissue and there was a decrease in tumor diameter. Our findings indicate that radio frequency hyperthermia has a beneficial effect in the treatment of malignant glioma.

Keywords: minimally invasive techniques, deep seated brain tumors, radio frequency hyperthermia, glioma, necrosis, CT, MRI.

\section{Corresponding author:}

Name : Mohamed Salah Mohamed Ahmed

Tel: 01095390159

\section{INTRODUCTION}

$\mathbf{H}$ igh grades brain tumors, such as glioblastoma and anaplastic astrocytoma, are regarded as challenges for neurosurgeons ${ }^{1}$. Glioma accounts for $25-33 \%$ of all brain tumors and usually relapses very quickly after surgery ${ }^{2}$. Patients with grade IV glioma generally relapse within 3 months after surgery and tumor size can return to presurgery size by 6 months after surgery ${ }^{3}$. Without treatment, malignant glioma patients only survive for an average of 17 weeks, and life span can be prolonged to 30 weeks after surgery and chemotherapy. Patients with recurrent malignant glioma repeatedly receive craniotomy at short time intervals, which is not only a severe physical burden but also a heavy economic cost for both family and society ${ }^{4}$.Though, neurosurgeon need a new treatment modalities that has a low impact on patients and can be repeated multiple times at low cost. Radio frequency ablation treatment is a sub-stantive tumor therapy. Surgical, radiotherapy and chemotherapy treatments for malignant brain tumors are unsatisfactory; so it is a need to find a new therapeutic approach. Malignant glioma is very sensitive

\section{Email : msm_1016@yahoo.com}

to heat. Therefore, malignant brain tumor heat treatment could potentially be used as a new modality. Radiotherapy and chemotherapy have proved to be suboptimal modalities in the treatment of malignant brain tumors. Thermal ablation has received increasing attention from clinicians as a new alternative treatment ${ }^{\mathbf{5 , 6 , 7}}$.In our study, we used a brain tumor hyperthermia apparatus to treat malignant glioma patients, and subsequently performed medical imaging examinations to observe efficacy. The results of treatment are as follows.

\section{Design}

SUBJECTS AND METHODS

Intervention clinical study

\section{Time and setting}

The study was performed between 2015 and 2017 in Neurosurgery Department at Zagazig University Hospitals.

\section{Subjects}

Fifteen patients were recruited from neurosurgery department at Zagazig University Hospitals between 2015 and 2017, who had grade II, III and IV primary or recurrent malignant gliomas (tumor diameter 2-5 cm)according to the WHO 
classification[1]and being given a full explanation of the possible side effects and benefits of hyperthermia.

Inclusion criteria:- patients with:

- Tumor size $2-5 \mathrm{~cm}$ in diameter.

- metastatic tumors.

- recurrent tumors.

- Subcortical brain tumors more than $2 \mathrm{~cm}$ or deep structures tumors (basal ganglia, thalamus and interventricular tumors)

- Patient's understanding and acceptance of the type of procedure.

- Patient's consent to participate in study.

\section{Methods}

Complete system for neurosurgical ablation and lesioning, including Radiofrequency mode. Complete with cables and remote control. Electrodes need to be specified separately. This version of Leksell@ Neuro Generator provides significant enhancements: non pulsed RF and Active Tissue Characterization. The RF feature reduces side-effects considerably when performing ablation treatmentstereotactic guided CT scan assisted stereotactic apparatus to insert the electrode needle into the center of tumors. The electrode needles could be placed together. The positions of the electrode needles were adjusted using the pre-operation simulated temperature distribution model. Temperature should be constantly with the tumor periphery temperature maintained at $42-43^{\circ} \mathrm{C}$ for approximately 5 minutes.

\section{RESULTS}

Quantitative analysis of participants

Fifteen patients with brain tumours (primary and recurrent) were included in the study and received hyperthermia in form of radiofrequency. Patients with grade III-IV primary glioma did not receive concurrent chemotherapy and radiotherapy, while recurrent glioma patients underwent radiotherapy and chemotherapy) received hyperthermia. All 15 patients were involved in the final analysis. The baseline information for the 30 patients enrolled in the study is detailed in Table 1.

\begin{tabular}{|c|c|c|c|c|c|c|}
\hline $\begin{array}{l}\text { Case } \\
\text { no }\end{array}$ & Sex & Age(years) & Type & Grade & Diameter $(\mathrm{cm})$ & Treatment \\
\hline 1 & male & 52 & Metastatic & metastatic & 2.5 & $\mathrm{H}+\mathrm{R}$ \\
\hline 2 & male & 82 & primary & IV & 3 & $\mathrm{H}+\mathrm{R}$ \\
\hline 3 & female & 34 & primary & III & 3.2 & $\mathrm{H}$ \\
\hline 4 & male & 6 & primary & II & 1.5 & $\mathrm{H}$ \\
\hline 5 & female & 7 & recurrent & II & 3.8 & $\mathrm{H}$ \\
\hline 6 & female & 20 & primary & II & 2 & $\mathrm{H}$ \\
\hline 7 & male & 39 & primary & III & 2 & $\mathrm{H}$ \\
\hline 8 & female & 55 & primary & II & 4 & $\mathrm{H}$ \\
\hline 9 & male & 45 & primary & II & 2 & $\mathrm{H}$ \\
\hline 10 & male & 65 & primary & IV & 2 & $\mathrm{H}+\mathrm{R}$ \\
\hline 11 & Female & 36 & primary & III & 2 & $\mathrm{H}$ \\
\hline 12 & Female & 55 & primary & II & 2.5 & $\mathrm{H}$ \\
\hline 13 & Female & 50 & primary & III & 3 & $\mathrm{H}$ \\
\hline 14 & Male & 58 & primary & II & 2 & $\mathrm{H}$ \\
\hline 15 & Female & 39 & primary & II & 2 & $\mathrm{H}$ \\
\hline
\end{tabular}


Table (2): Pre and Post-operative tumor size among the studied group underwent radiofrequency:

\begin{tabular}{|l|c|c|c|c|}
\hline \multicolumn{1}{|c|}{ Variable } & $\begin{array}{c}\text { Pre-operative } \\
(\mathbf{n = 1 5})\end{array}$ & $\begin{array}{c}\text { Post-operative } \\
(\mathbf{n = 1 5})\end{array}$ & Test & P \\
\hline $\begin{array}{l}\text { Tumor size: } \\
\text { Mean } \pm S D\end{array}$ & $6.84 \pm 3.54$ & $3.92 \pm 2.65$ & $\mathbf{- 3 . 2 9 9}$ & $\mathbf{0 . 0 0 1}$ \\
$\begin{array}{l}\text { Median } \\
\text { Range }\end{array}$ & 6 & 3.9 & & (S) \\
\hline
\end{tabular}

This table shows that there was significant difference between pre and postoperative size of the tumor with the tumor's size was significantly lower in postoperative than in preoperative assessment (3.92 vs 6.84 respectively).

\section{Effects of hyperthermia}

Patients underwent head CT or MRI examination at 3 months after treatment to monitor

changes in tumor size and tumor growth slowed or stopped and the tumor had low density. The heated centers of $80 \%$ of the tumors presented with necrosis, intracranial hypertension symptoms were improved and life expectancy was prolonged. Thus, the results indicated that hyperthermia was effective in the treatment of malignant glioma.

\section{Analysis of representative case}

Case 1 was a 45 year-old man (No. 9 in Table 1). He received radiofrequency ablation treatment on remained in good health during a long-term follow-up period up till now he still good with no neurological deficit in fig $(1,2,3)$

\section{Side effects}

Adverse reactions including early induced hemorrhage and neeuorolical deficit may aggravate cerebral edema a short time after treatment.

\section{DISCUSSION}

In last decades, conventional methods of treatment for this disease have failed to control tumor progression ${ }^{8,9}$. In basic and clinical research studies a new treatment modality, hyperthermia, has shown remarkable effectiveness in killing neoplastic cells. Primary glioma patients account for about one third of all brain tumors, and there are also many recurrent patients. Selecting patients for procedure.

The effects of hyperthermia on glioma can be determined in a few days or weeks after treatment. Hyperthermia is a safe treatment involving a low power, slow heating process and minimal damage to surrounding normal tissue. It also results in little discomfort to the patient. The treatment efficacy of hyperthermia is superior to that of laser, freezing and tumor immunotherapy ${ }^{\mathbf{1 0 , 1 4}}$. The center of tumors can be heated to a temperature $\geq 43^{\circ} \mathrm{C}$. However, there are normal neurons located around the periphery of the tumor, so the temperature in this region should be maintained at $\leq 43^{\circ} \mathrm{C}$ to avoid side effects $^{12,13}$. Heat can block tumor cell respiration, decrease oxygen consumption and reduce the $\mathrm{pH}$ in the extracellular environment, thus inhibiting cell proliferation $^{11}$. and can enhance the activity of cellular lysosomes and promote lysozyme function which damages mitochondria, the Golgi apparatus and other organelles, leading to tumor cell destruction ${ }^{\mathbf{1 5}}$.

Hyperthermia treatment for brain tumors utilizes a CT scan assisted stereotactic apparatus to insert the electrode needle into the center of tumors. Two electrode needles can be placed together. In the present study, thermometry catheters, which are fine soft tubes, were inserted around the tumor periphery. The positions of the electrode needles were adjusted using a pre-operation simulated temperature distribution model. Temperature around tumor periphery kept around $42-43^{\circ} \mathrm{C}$. Stereotactic surgery is simple, convenient and minimally invasive, with short surgery time and can be performed under local anesthesia. Hyperthermia was carried out in the ward. Patients experience no heat, pain or headaches. The treatment rarely 
has complications and can be completed within 1 hour. One advantage of hyperthermia is its minimal invasiveness; it can be carried out with no complications on inoperable patients with systemic failure, on elderly patients with poor cardiopulmonary function, and on patients with tumors in eloquent areas of brain in which surgery can lead to disability. In the current study, the survival rate of glioma patients was not the main Case (1)
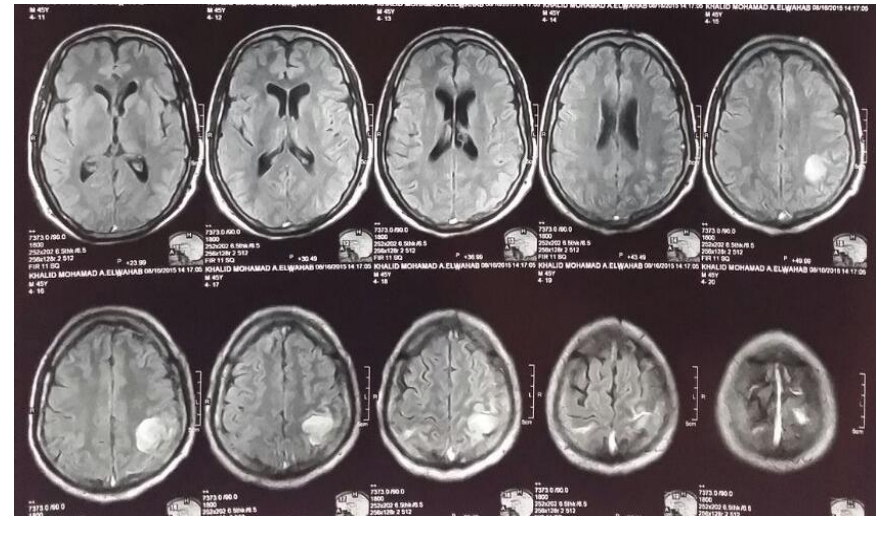

fig (1 ): male patient 45 years old presented with headache vomiting with $R T$ side weakness. Radiologically mri brain flair image and $T 2$ image showing left parietal hyper intense round lesion with no edema measure $2 \times 2 \mathrm{~cm}$ operated for stereotactic biopsy and radiofrequency ablation.
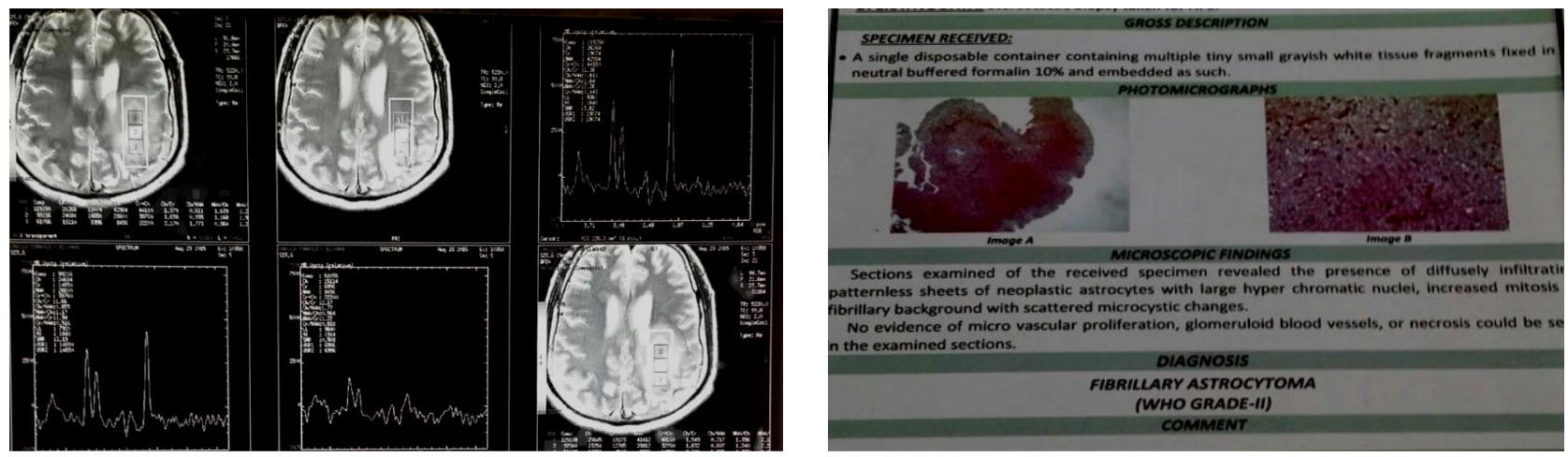

Fig (2): MRS for brain lesion showing choline peaking most probably brain tumor histopathology of brain tumor stereotactic guided biopsy showing glioma grade ii.
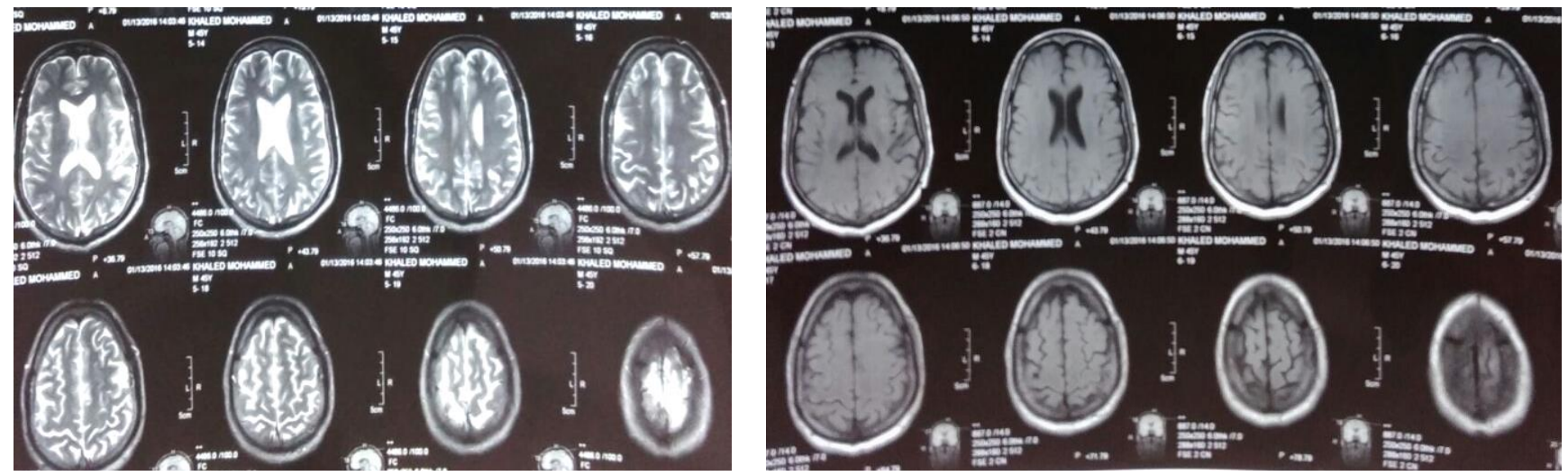

Fig(3) : follow up MRI brain of patient operated for stereotactic guided biopsy and radiofrequency ablation 
for the lesion after 3 month showing reduction of the lesion size with no residual can be seen in this follow up mri

\section{CONCLUSION}

Radiofreuency ablation therapies provide a minimal invasive technique to cancer therapy that is gaining clinical acceptance. In this study has revealed a good outcome in most of the thermal ablation., Thermal ablative techniques may provide a palliative option and the ability to treat patients who are not surgical candidates. This technique may provide multiple advantages. First, it is minimally invasive and compatible with stereotactic surgery especially for the treatment of deep-seated tumors that are usually inaccessible for conventional surgery. This avoids the risk of morbidity associated with open craniotomy and permits for more rapid patient recovery and a shorter hospital stay. Second, under local anesthesia while patients are awake during the procedure.

\section{REFERENCE}

1. Ahmed K, Zaidi SF Treating cancer with heat: hyperthermia as promising strategy to enhance apoptosis. J Pak Med Assoc 2013.,63: 504-508

2. Ahmed M, Goldberg SN. Basic science research in thermal ablation. Surg Oncol Clin N Am.2011 Apr;20(2):237-58.

3. Bath $\mathbf{C}$ :Using hyperthermia for cancer treatment: proofs, promises, and uncertainties. 2014 ASCO Post.

4. Bergman JJ, Zhang YM, He S, Weusten B, Xue L, Fleischer DE, Lu N, Dawsey SM, Wang GQ. Outcomes from a prospective trial of endoscopic radiofrequency ablation of early squamous cell neoplasia of the esophagus. Gastrointest Endosc. 2011;74(6):1181-90.

5. Bhardwaj N, Strickland AD, Ahmad F, Atanesyan L, West K, Lloyd DM. A comparative histological evaluation of the ablations produced by microwave, cryotherapy and radiofrequency in the liver. Pathology 2009; 41: 168-72.

6. Fabiano AJ, Qiu J. Delayed failure of laserinduced interstitial thermotherapy for postradiosurgery brain metastases. World Neurosurg. 2014;82(3-4):e559-563

7. Ferlay, J., Soerjomataram, I., Ervik, M., Dikshit, R., Eser, S., Mathers, C., Rebelo, M., Parkin, D.M., Forman, D., Bray, F., GLOBOCAN 2012 v1.0, Cancer Incidence and Mortality Worldwide: IARC Cancer Base No. 11 [Internet].,2013 International Agency for Research on Cancer, Lyon, Franc.

8. Frey B, Weiss EM, Rubner Y, Wunderlich R, Ott OJ, : Old and new facts about hyperthermia-induced modulations of the immune system. Int J Hyperthermia 2012.,28: 528-542

9. Gao S, Zheng M, Ren X, Tang Y, Liang X: Local hyperthermia in head and neck cancer: mechanism, application and advance. Oncotarget 2016., 7: 57367-57378.

10.Garrett M, Consiglieri G, Nakaji P : Transcranial minimally invasive neurosurgery for tumors. Neurosurg Clin $\mathrm{N}$ Am 2010.,21:595-605, v.

11.Gassino R, Liu Y, Konstantaki M : A fiber optic probe for tumor laser ablation with integrated temperature measurement capability. Journal of Light wave Technology 2017 35(16): 3447-3454.

12.jiahang Sun, Mian Guo, Hengyuan Pang, Jingtao Qi, Jinwei Zhang, and Yunlong Ge: Treatment of malignant glioma using hyperthermia Neural Regen Res. Oct 15, 2015; 8(29): 2775-2782

13.Shi Y, Mao Y (2017) Magnetc resonance thermometry guided laser intersttal thermal therapy in neurosurgery, a promising tool for dural-based lesions? World Neurosurgery 98: 836-838.

14.Siegel RL, Miller KD, Jemal A (2016) Cancer statstcs, 2016. CA Cancer J Clin 66: 730.

15.Sonntag PD, Hinshaw JL, Lubner MG, Brace CL, Lee FT Jr. Thermal ablation of lung tumors. Surg Oncol Clin N Am. 2011;20(2):369-87. 